\title{
Who Is to Blame for False-Negative Sentinel Node Biopsies in Melanoma?
}

\author{
Vernon K. Sondak, $\mathrm{MD}^{1,2}$ and Jonathan S. Zager, $\mathrm{MD}^{1,2}$ \\ ${ }^{1}$ Department of Cutaneous Oncology, Moffitt Cancer Center, Tampa, FL; ${ }^{2}$ Departments of Oncologic Sciences and \\ Surgery, University of South Florida College of Medicine, Tampa, FL
}

No matter how one defines standard of care, sentinel lymph node biopsy (SLNB) has become standard of care for the treatment of clinically localized melanoma. ${ }^{1,2}$ Given this fact, it is surprising that more attention has not been paid to the issue of false-negative results of sentinel node biopsy. In the current issue of Annals of Surgical Oncology, Scoggins et al. report on the false-negative rate of sentinel node biopsy from a large series of patients entered prospectively into the multi-institutional Sunbelt Melanoma Trial. ${ }^{3}$ An important starting point for any discussion of this topic is how to define and report the falsenegative rate. Some investigators have reported the number of false-negative sentinel node biopsies (generally defined as any first recurrence of melanoma in a lymph node potentially draining the primary tumor) occurring out of all procedures performed. This is an incorrect representation of the false-negative rate and not a relevant number to patients and surgeons. In contrast, a very important number is the ratio of true-negative biopsies to all negative biopsies (false negatives plus true negatives). This is the negative predictive value of the sentinel node biopsy procedure and is of great value to surgeons when they counsel patients after a negative sentinel node biopsy procedure, but it is still not the false-negative rate. The negative predictive value of sentinel node biopsy in large series has been reported between 94 and $98.5 \%$ (Table 1), meaning that only 2-6 patients per 100 who are told their nodes are negative are given that information incorrectly. However, the actual definition of the false-negative rate is the ratio of false-negative results to the total number of positive lymph nodes (false negatives plus true positives). This value has

(C) Society of Surgical Oncology 2009

Published Online: 2 December 2009

V. K. Sondak, MD

e-mail: vernon.sondak@moffitt.org been reported in the range 6-21\%. Importantly, any method of reporting on the likelihood that a truly positive lymph node will be missed by the sentinel node procedure depends on the a priori likelihood that any lymph nodes are actually involved by melanoma. Put another way, if all the lymph nodes in a patient are uninvolved by tumor, it does not matter which nodes the surgeon removes-the result will be a true negative. The corollary of this, however, is that patients whose melanoma is most likely to have spread to the regional nodes are those most likely to have a falsenegative sentinel node biopsy. It also follows that different series of patients undergoing sentinel node biopsy using identical techniques (and with identical accuracy) will report different false-negative rates and negative predictive values if the baseline characteristics of the melanoma patients differ. In addition, because nodal recurrences can take many years to manifest clinically, as the duration of follow-up increases, the false-negative rate and total positive node percentage will increase. In the Sunbelt Melanoma Trial data reported by Scoggins et al., after median follow-up of 61 months, the overall likelihood of a positive lymph node was $19.8 \%(486 / 2,451)$, the negative predictive value of a sentinel node biopsy was $97.0 \%$ $(1,906 / 59+1,906)$, and the false-negative rate was $10.8 \%$ $(59 / 59+486){ }^{3}$ The corresponding values in other contemporary large series of melanoma patients undergoing sentinel node biopsy are shown in Table 1 .

So now let us ask the question: who is to blame for a false-negative sentinel node biopsy? Please note that, in this politically correct (and highly litigious) world, the word "blame" carries connotations that many surgeons would just as soon avoid. We use the word advisedly (and tonguein-cheek) in this editorial: in fact, we do not imply directly or indirectly that blame should truly be assigned each time a lymph node recurrence occurs after a prior sentinel node biopsy, nor do we suggest that anyone did anything wrong 
TABLE 1 Nodal recurrences after negative sentinel lymph node biopsy for melanoma in selected large series

\begin{tabular}{|c|c|c|c|c|c|c|}
\hline $\begin{array}{l}\text { Author (year } \\
\text { of publication) }\end{array}$ & $\begin{array}{l}\text { Number } \\
\text { of patients }\end{array}$ & $\begin{array}{l}\text { Median } \\
\text { follow-up } \\
\text { (months) }\end{array}$ & $\begin{array}{l}\text { Sentinel node } \\
\text { positivity rate, } \\
\text { TP/all cases }(\%)\end{array}$ & $\begin{array}{l}\text { Nodal recurrences } \\
\text { in sentinel node-negative } \\
\text { basin (\% of all patients) }\end{array}$ & $\begin{array}{l}\text { Negative } \\
\text { predictive } \\
\text { value }(\%)\end{array}$ & $\begin{array}{l}\text { False-negative } \\
\text { rate, FN/ } \\
(\mathrm{TP}+\mathrm{FN})(\%)\end{array}$ \\
\hline Chao $(2002)^{15}$ & 1183 & 16 & $233 / 1183(19.7 \%)$ & $14(1.1 \%)$ & 98.5 & 5.6 \\
\hline Testori $(2009)^{16}$ & 1313 & 54 & $220 / 1313(16.9 \%)$ & $36(2.7 \%)$ & 96.3 & 14.4 \\
\hline Morton $(2006)^{13}$ & 769 & 60 & $122 / 769(16 \%)$ & $26(3.3 \%)$ & 96.0 & 17.6 \\
\hline Nowecki (2006) ${ }^{17}$ & 1207 & 36 & 228/1207 (18.9\%) & $43(3.6 \%)$ & 94.2 & 20.0 \\
\hline Cascinelli $(2006)^{18}$ & 1108 & 61 & $176 / 1108(15.9 \%)$ & $47(4.2 \%)$ & 95.0 & 21.0 \\
\hline
\end{tabular}

FN false negative, $T P$ true positive

that led to an adverse outcome. However, there must be a reason why the sentinel node was reported as negative but the patient developed a nodal recurrence. So, offered in this spirit as looking for someone to blame, what are some possible reasons and what can we learn from them?

Certainly, we must start our discourse by looking to the surgeon. Are we removing all the correct node(s) each and every time we perform a sentinel node? Why would we not? Well, for starters, the operational definition of a sentinel node is itself somewhat arbitrary, particularly in terms of the amount of radioactivity that can be contained within a lymph node before it is deemed hot enough to remove. Just as arbitrary, if not more so, is the point at which a basin can be considered clear of residual sentinel nodes, that is, that any residual radioactivity in the basin represents background or shine-through from the primary tumor site. Because of the potential for shine-through to mask a small retained sentinel node, we might well expect higher rates of false-negative results the closer the primary tumor is to the sentinel node, but with the possible exception of upper outer quadrant breast cancer primaries, this has never been documented or even directly studied. ${ }^{4}$

However, the sentinel node biopsy procedure begins in Nuclear Medicine, so what can we learn by looking there? The nuclear medicine technologist has to make sure that the injection is performed correctly with the $\mathrm{Tc}^{99}$ sulfur colloid injection as close to the scar or biopsy site as possible and in such a way that drainage from that site truly recapitulates the actual dermal lymphatic drainage of that particular area of skin. Deeper injection may reduce the accuracy of identifying true sentinel nodes, as would injection farther away from the biopsy site. A sobering study involved patients undergoing lymphoscintigraphy at the same site on two separate occasions: the same nodes were not always identified on both studies, suggesting that minor technical variations could result in some sentinel nodes being missed. ${ }^{5}$ Even if the correct node accumulates the radiotracer, ascertaining the location of that node (or even the basin in which it resides) may be inaccurate, especially in the head and neck. Certainly, there are numerous reports to suggest that higher false-negative rates are associated with head and neck primary sites. ${ }^{6}$ A potential solution could be the use of single-photon emission computed tomography with computed tomography scanning (SPECT/CT) lymphoscintigraphy imaging, which has been shown to increase the resolution and improve anatomic localization for sentinel nodes in the head and neck. Van der Ploeg and colleagues showed the potential value of SPECT/CT lymphoscintigraphy: in a series of 85 patients who underwent both conventional lymphoscintigraphy and SPECT/CT imaging prior to SLNB, SPECT/CT resulted in a different incision in 17 patients, an incision at another site in 8 , and an extra incision in 5 patients. ${ }^{7}$ SPECT/CT identified 12 nodes not seen on standard scanning (an extra 8\%). Ten of these 12 nodes were harvested and 2 contained lymphatic metastases. This certainly provides strong evidence that better lymphoscintigraphy could lower the false-negative rate, especially for our most difficult sites such as the head and neck.

Can the pathologist be to blame for false-negative results in certain cases where the correct node has been identified and removed? Routine examination of the sentinel node includes immunohistochemical staining techniques using antibodies to melanocyte lineage antigens, which while very sensitive, is certainly not foolproof. Even with the intensive attention that the sentinel node receives, only a small portion of the entire lymph node is examined. Hence it is entirely possible that the pathologist may not see the tumor cells in a sentinel node and call it negative. Those patients would not undergo completion node dissection and hence would be at risk of failure in nonsentinel nodes, and these cases would be considered false-negative results. Evidence suggests that more intensive sampling of the sentinel node does indeed identify more positive nodes, but as yet there is no proof that this more time-consuming approach decreases false-negative results. ${ }^{8}$ As we perform sentinel node biopsies for thinner melanomas (our routine is to recommend the procedure for 
patients with melanomas $\geq 0.76 \mathrm{~mm}$ ), the chances of very small tumor deposits or even isolated tumor cells being the only metastases present in the node increases, and the challenges for the pathologist increase accordingly. In a paper by Scheri and colleagues examining the impact of micrometastases $(\leq 0.2 \mathrm{~mm})$ and isolated tumor cells in the sentinel node, the authors found that the incidence of positive nonsentinel nodes in the completion node dissection specimen was $12 \% .^{9}$ In addition, the 5-year melanoma-specific survival rate for those patients with isolated tumor cells or very small metastatic foci when compared with those who were deemed sentinel node negative was significantly lower (94 vs. $89 \%, p=0.02$ ). Future studies on more intensive analysis of sentinel nodes, including molecular analysis using reverse-transcriptase polymerase chain reaction (RT-PCR) technology, should look at whether these techniques can decrease the falsenegative sentinel node biopsy rate while also taking into account the potential risk that some of these techniques, especially RT-PCR, may be identifying false-positive cases. ${ }^{10}$

One potential issue that has only received limited attention is the potential that the initial melanoma biopsy could sufficiently disrupt lymphatic drainage to result in false-negative results (meaning we could blame dermatologists and primary-care practitioners for failures). Melanomas are diagnosed by biopsies that range from punch and incisional biopsies that leave much of the lesion intact to the more common shave, scallop, and excisional biopsies that remove all grossly evident lesion. Is there any evidence that, after a biopsy that removes the entire lesion, the draining lymphatics are sufficiently disrupted that the accuracy of the sentinel node biopsy procedure could be compromised? Taken to the extreme, Kelemen et al. demonstrated that, while sentinel node biopsy is feasible after a previous wide excision, the false-negative rate does in fact go up. ${ }^{11}$ However, to attempt to address the biopsy issue, Trifiro et al. identified 31 patients with lesions that were clinically considered to represent definite melanomas prior to any type of biopsy, and had them undergo lymphoscintigraphy before and after excisional biopsy. In 23 of 31 cases the scans were concordant, and in 6 cases new basins were seen after excisional biopsy was performed. However, in two cases, nodal basins initially identified as draining the primary site were not seen on the postexcision scan. ${ }^{12}$ Although there were no false-negative cases reported by those authors, their data provide evidence that clinically appropriate and necessary biopsies may at times disrupt lymphatic channels sufficiently to influence the accuracy of sentinel node biopsy, and further studies are warranted to identify which, if any, primary melanomas should best be diagnosed by nonexcisional biopsy procedures to minimize this effect.
Lastly, it may not always be any physician's fault. There could be immutable patient-related factors that lead to some irreducible minimum number of false-negative sentinel node biopsies, no matter how well every other aspect of the patient's care has proceeded. For example, it may be that, at time of diagnosis and sentinel node biopsy, there are already metastatic tumor cells within the lymphatic channels that simply have not made it all the way to the node yet. There is no actual evidence for or against this, and indeed the MSLT-1 randomized trial did not find any evidence that sentinel node biopsy increased the likelihood of in-transit metastasis. ${ }^{13}$ However, we do have evidence of declining lymphatic dysfunction with age, which may be a potential factor in some false-negative sentinel node biopsies. Conway et al. have shown that lymphatic function, as assessed by radiocolloid transit to and uptake within the sentinel node, declines with age. ${ }^{14}$ This putative age-related lymphatic dysfunction could be due to limitations of transit of lymph from the primary site to the node, or it could be due to diminished filtration function of the aging lymph node, with greater pass-through to secondechelon nodes. Either of these, but particularly the latter, could increase false-negative sentinel node biopsy rates in older patients. Intriguingly, Scoggins et al. found that older patients were more likely to have false-negative results than were younger patients. ${ }^{3}$

So if false-negative sentinel node biopsy results are likely to be with us for a while, even if we do everything right, what are the consequences for our patients? Are they worse off having a false-negative sentinel node biopsy than if they had not undergone the procedure at all? The MSLT1 randomized trial allowed for a comparison between patients with a false-negative biopsy, with a $68.4 \%$ relapse rate at 3 years, and those in the observation group who relapsed in the nodes without a prior sentinel node biopsy $(64.9 \%$ at 3 years, $p=0.60) .{ }^{13}$ In the Sunbelt Melanoma Trial results reported in this issue, which did not have a nodal observation arm, the overall survival of patients with false-negative sentinel node biopsy results was not statistically significantly worse than those with true-positive sentinel nodes. ${ }^{3}$

This is encouraging news that should reassure that small percentage of patients who do return with a palpable node months or years after a negative sentinel node biopsy. However, is there anything we can or should be doing to detect recurrences earlier in patients at highest risk? First, who are these patients? Certainly, patients with thick, ulcerated, and/or high-mitotic-rate primaries are at high risk for false-negative results, because they are at high risk of having a positive node in the first place. Furthermore, it seems that older patients, patients with head and neck primaries, and patients undergoing sentinel node biopsy after prior wide excision are groups at increased risk of 
false-negative biopsy. Perhaps we can even include patients with discordant results between their lymphoscintigraphy and the findings at sentinel node biopsy (e.g., those in whom the scan shows three nodes but the sentinel node procedure only yields two) in this group, although that is speculative at this point. So what, if anything, should we do to monitor high-risk patients? The MSLT-2 trial is prospectively evaluating whether nodal ultrasound can help monitor nodal basins that are known to be sentinel node positive but that do not undergo complete dissection. If so, nodal ultrasound for surveillance of patients considered at risk of false-negative sentinel node biopsy results would potentially make sense. Some European centers with familiarity with ultrasonography already do this, and as we have become more comfortable with nodal ultrasound at our institution, we are employing it selectively in our highrisk sentinel-node-negative patients. However, prospective evaluation is warranted before this approach is widely adopted.

So where does this leave us? Nodal recurrences after prior negative sentinel node biopsy now account for a significant percentage of all clinical stage III melanoma patients seen in multidisciplinary clinics such as our own. Improving the accuracy of sentinel node biopsy should be an active area of research, and efforts to identify and monitor patients at risk of nodal recurrence after negative biopsy seem warranted as well. It is time that this important issue received the attention it deserves.

\section{REFERENCES}

1. Strauss DC, Thomas JM. What does the medical profession mean by "standard of care?" J Clin Oncol. 2009; Epub ahead of print.

2. Balch CM, Morton DL, Gershenwald JE, et al. Sentinel node biopsy and standard of care for melanoma. J Am Acad Dermatol. 2009;60:872-5.

3. Scoggins CR, Martin RCG, Ross MI, et al. Ann Surg Oncol. 2009; this issue.

4. Krag D, Weaver D, Ashikaga T, et al. The sentinel node in breast cancer - a multicenter validation study. N Engl J Med. 1998;339: 941-6.
5. Uren RF, Howman-Giles R, Chung DK, et al. The reproducibility in routine clinical practice of sentinel lymph node identification by pre-operative lymphoscintigraphy in patients with cutaneous melanoma. Ann Surg Oncol. 2007;14:899-905.

6. Carlson GW, Page AJ, Cohen C, et al. Regional recurrence after negative sentinel lymph node biopsy for melanoma. Ann Surg. 2008;248:378-86.

7. Van der Ploeg IM, Valdes Olmos RA, Kroon BB, et al. The yield of SPECT/CT for anatomical lymphatic mapping in patients with melanoma. Ann Surg Oncol. 2009;16:1537-42.

8. Murali R, Cochran AJ, Cook MG, et al. Interobserver reproducibility of histologic parameters of melanoma deposits in sentinel lymph nodes: implications for management of patients with melanoma. Cancer. 2009;115:5026-37.

9. Scheri RP, Essner R, Turner RR, et al. Isolated tumor cells in the sentinel node affect long-term prognosis of patients with melanoma. Ann Surg Oncol. 2008;14:2861-6.

10. Scoggins CR, Ross MI, Reintgen DS, et al. Prospective multiinstitutional study of reverse transcriptase polymerase chain reaction for molecular staging of melanoma. J Clin Oncol. 2006;24:2849-57.

11. Kelemen PR, Essner R, Foshag LJ, et al. Lymphatic mapping and sentinel lymphadenectomy after wide local excision of primary melanoma. J Am Coll Surg. 1999;3:247-52.

12. Trifiro G, Verrecchira F, Soteldo J, et al. Modification of lymphoscintigraphic sentinel node identification before and after excisional biopsy of primary cutaneous melanoma. Melanoma Res. 2008;18:373-7.

13. Morton DL, Thompson JF, Cochran AJ, et al. Sentinel-node biopsy of nodal observation in melanoma. New Engl J Med. 2006;13:1307-17.

14. Conway WC, Faries MB, Nicholl MB, et al. Age-related lymphatic dysfunction in melanoma patients. Ann Surg Oncol. 2009; $16: 248-52$.

15. Chao C, Wong SL, Ross MI, et al. Patterns of early recurrence after sentinel lymph node biopsy for melanoma. Am J Surg. $2002 ; 18: 520-4$.

16. Testori A, De Salvo GL, Montesco MC, et al. Clinical considerations on sentinel node biopsy in melanoma from an Italian multicentric study on 1313 patients (SOLISM-ILI). Ann Surg Oncol. 2009;16:2018-27.

17. Nowecki ZI, Rutkowski P, Nasierowska-Guttmejer A, et al. Survival analysis and clinicopathological factors associated with false-negative sentinel lymph node biopsy findings in patients with cutaneous melanoma. Ann Surg Oncol. 2006;13:1655-63.

18. Cascinelli N, Bombardieri E, Buffalino R, et al. Sentinel and nonsentinel node status in stage IB and II melanoma patients: two-step prognostic indicators of survival. J Clin Oncol. 2006;24:4464-71. 